\title{
The Contribution of Interference Control for Young Children`s Working Memory Performance: Insights from Eye-tracking
}

\author{
Claudia M. Roebers \\ University of Bern \\ Corinne Schmid \\ Thomas Roderer \\ University of Bern \\ University of Bern
}

\begin{abstract}
In the present study, the role of visual attentional processes for working memory performance in a sample of 6-year-olds was investigated. This was done by combining an individual differences approach with an experimental manipulation: For the individual differences approach, participants were grouped based on their performance in a classical interference control task, and their working memory skills were systematically compared. For the experimental manipulation, the need to control interference while performing a working memory task was increased in one condition through presentation of distracting stimuli. In a between-subject design performance in this condition was contrasted with a control condition without distractors. Additionally, fixation time during stimuli presentation were quantified by tracking participants' gazes. Results revealed that children with higher interference control skills showed superior working memory performance. Increasing the need to inhibit attention towards task-irrelevant information through presentation of distractors decreased working memory performance. The present study offers supporting evidence for a close relationship between young children`s working memory and attention.
\end{abstract}

Keywords: Working memory, interference control, attentional control, fixation time, children

\section{Introduction}

Working memory is a central theoretical construct in cognitive psychology. Although there are different theoretical conceptualisations of working memory in the literature, most researchers agree that limited capacity in working memory is a key aspect (Baddeley, 2001; Cowan, 2005; Kane \& Engle, 2000, 2003). Working memory skills play an important role for a range of higher-order cognitive functions throughout the lifespan (for a recent overview see Cowan \& Alloway, 2009). In the context of learning and academic achievement, individual differences in working memory predict writing performance, vocabulary acquisition, and arithmetic skills (e.g., Bull, Johnston, \& Roy, 1999; Gathercole, Alloway, Willis, \& Adams, 2006; Gathercole \& Baddeley, 1993; Lee, Ng, \& Ng, 2009; St ClairThompson \& Gathercole, 2006; Swanson \& Kim, 2007). In addition, working memory has been found to account for substantial amounts of variance in central aspects of cognition like general fluid intelligence, reasoning or reading comprehension (e.g., Daneman \& Merikle, 1996; Kyllonen \& Christal, 1990; Engle, Tuholski, Laughlin, \& Conway, 1999). Importantly, studies have consistently shown that working memory tasks requiring participants to retain information for immediate recall, to maintain and/or update information, and to simultaneously process additional aspects of the task, constitute better predictors for achievement and general intelligence than, for example, short-term memory capacity (e.g., Bayliss, Jarrold, Gunn, \& Baddeley, 2003; Cowan \& Alloway, 2009; Engle et al., 1999; Gathercole et al., 2006; Kail \& Hall, 2001).

Contemporary theoretical frameworks of working memory embrace aspects of attention such as taskswitching (Towse, Hitch, \& Hutton, 1998), focus of attention (Cowan et al., 2005), or controlled attention (Kane \& Engle, 2003). In other words, it appears that 
selectively attending to task-relevant information and maintaining this information in working memory while, at the same time, inhibiting attendance and processing of task-irrelevant information is crucial for mastering classical working memory tasks. Although the theoretical perspectives differ in terms of which underlying attentional processes contribute to which aspects of working memory and what the exact underlying mechanisms for this relationship are, it seems clear that an individual's ability to control interference (stemming, for example, from a need to continuously switch attention between targets, to update information to prevent memory trace decay, and/or from the need to inhibit attention towards distractors) substantially influences working memory performance (Cowan, 2005; Kane \& Engle, 2003; Baddeley, 2001).

While a relation between working memory and interference control skills seems to be well established for adults and school-aged children, much less is known about this association in even younger children. For 5and 6-year-olds' developing school readiness, interference control skills are currently being discussed as developmental antecedents of working memory growth (Best, Miller, \& Jones, 2009; Garon, Bryson \& Smith, 2008): young children's interference control skills may have to be developed to a certain degree before substantial increases in working memory capacity can be expected. Supporting empirical evidence for this relationship between working memory and interference control in young children, however, is still rare. The present study aimed at exploring whether interference control and working memory are related in 6-year-old children.

Investigations of the assumed interference control working memory relation can, on the one side, be found in the field of general and cognitive psychology. In this line of research, individuals with high versus low interference control skills are systematically compared in terms of their working memory capacity, typically under conditions of high versus low distraction and experimentally induced interference (Kane, Conway, Hambrick, Engle, 2008; Kane \& Engle, 2000; 2003). On the other side, researchers interested in scene perception (Hollingworth \& Henderson, 2002; Võ, Schneider, \& Matthias, 2008; Wedel, Pieters, \& Liechty, 2008), visual feature search (Wienrich, Hesse, \& Müller-Plath, 2009), or other domains involving visual attention, working memory and memory (Awh \& Jonides, 2001), choose a different methodological approach for investigating potential interference control-memory-interactions. In this domain, eye-tracking technology has been established as a tool for quantifying visual information processing and attention allocation and has produced many and interesting insights. Especially for the investigation and/or inducement of attentional shifts, either top-down and voluntary or bottom-up and stimulus-driven, recording individuals' fixations and/or saccades has repeatedly proven to be a suitable research tool (for a review on eye-tracking research in developmental psychology, see Karatekin, 2007).

The present study combined the two abovementioned methodologies for studying the contribution of interference control processes in young children's working memory performance. In a between-subject design, half of the participants were confronted with a classical developmental working memory task (Pickering \& Gathercole, 2001; Hasselhorn et al., 2011). The other half of the participants was confronted with distracting stimuli while performing the working memory task. Presentation of distracting stimuli increased the need of interference control while the to-be-remembered stimuli were shown and processed. Participants' gazes were recorded simultaneously via eyetracking. This allowed to check the effectiveness of the experimental manipulation and to assess the amount of attention allocation towards target stimuli under conditions of normal versus experimentally induced increased distraction. If interference control is linked to working memory performance, children should perform worse in the condition with distractors compared to the condition without additional irrelevant stimuli. Moreover, children assiged to the condition with distractors should allocate less gaze time on the working memory target stimuli than children in the control condition.

Results from this approach can be important both from a theoretical and an applied perspective: Theoretically, a better understanding of the interference control-working memory interactions can lead to advances in the theoretical frameworks of human working memory in general. Moreover, insights into early stages of this relationship may deepen our appreciation of interference control skills contributing to developmental progression in various cognitive domains (Best et al., 2009; Garon et al., 2008). From an applied 
perspective and given that working memory skills are predictive for school readiness and school achievement (Alloway, 2007; Alloway, Gathercole, Willis, \& Adams, 2004; Gathercole et al., 2006; Gathercole \& Baddeley, 1993), knowledge about interference control - working memory-relations may be useful for early recognition and diagnosis of developmental risks, and for tailoring effective interventions. Ultimately, trainings that take these interrelations into account and systematically build up both aspects rather than focusing on only one aspect may arise from this line of research (Rueda, Rothbart, McCandliss, Saccomanno, \& Posner, 2005; Thorell, Lindqvist, Nutley, Bohlin, \& Klingberg, 2009).

In order to provide more and detailed evidence for a relation between working memory and interference control in young children, an individual differences approach was also included in the study: interference control skills were used as individual differences variable. That is, based on a task measuring interference control skills, participating children were grouped together and working memory performance was compared across groups of children with low, medium low (below average), medium high (above average), and high interference control skills. If interference control is related to young children's working memory skills, then working memory performance should differ between the four groups. Recent research has shown that in young children, processes of active goal maintenance and the amount of conflict are important and additional aspects of developing interference control skills (Davidson, Amso, Anderson, \& Diamond, 2006; Marcovitch, Boseovski, \& Knapp, 2007). Therefore, the adapted and childappropriate version of the original Flanker task (Eriksen $\&$ Eriksen, 1974) used in the present study not only comprised a high degree of conflict (one third of the trials were incongruent trials) but also called for active goal maintenance since the conflict trials were randomly intermixed with distracting trials in an unforeseeable sequence.

\section{Method}

\section{Participants}

A total of 75 children completed both tasks and were included in the final sample. Data from an additional 4 children were not included in the analyses because they were absent on one of the two testing occasions, had vision impairments (strabismus) or because eye tracking was poor (restless participants, thick glasses or technical failures). Based on performance in the interference control task, children were classified as either low, medium low, medium high, or high in interference control skills using the quartiles of the obtained performance distribution. With the constraint of an approximately even gender distribution, children within the interference control groups were then randomly assigned to one of the two between-subjects conditions. Table 1 gives an overview of the sample, the grouping and the assignments to the two experimental conditions. Importantly, as can be seen in Table 1, there were no systematic differences in chronological age between the groups and the experimental conditions.

\section{Table 1}

Demographic information as a function of group (based on performance in the interference control task (IC); quartiles)

\begin{tabular}{lcccc} 
& Low IC & $\begin{array}{c}\text { Medium } \\
\text { low IC }\end{array}$ & $\begin{array}{c}\text { Medium } \\
\text { high IC }\end{array}$ & High IC \\
\hline N overall & 19 & 19 & 19 & 18 \\
N in condition with distractors & 10 & 8 & 11 & 8 \\
N female & 6 & 14 & 9 & 9 \\
Mean Age in Months (SD) & $76.0(4.9)$ & $74.5(5.5)$ & $74.6(5.9)$ & $74.8(6.5)$ \\
\hline
\end{tabular}

A total of 38 children (19 girls and 19 boys) with a mean age of 6 years and 3 months (SD $=5$ months) were assigned to the experimental condition without distractors and 37 children (19 girls and 18 boys) with a mean age of 6 years and 3 months ( $\mathrm{SD}=6$ months) were assigned to the experimental condition with distractors. Participants were recruited from public kindergarten in the vicinity of Lucerne, Switzerland. Only children whose parents gave their written informed consent were included in the study. Power analysis were conducted using the G*Power program (Faul, Erdfelder, Lang, \& Buchner, 2007) and revealed that in this sample the likelihood of detecting large main effects for the interference control grouping or for the experimental manipulation of the WM task was $81 \%$ and $92 \%$ (with the level of significance being set to $5 \%)$.

\section{Procedure}

Participants were tested individually in a quiet room at their kindergarten. All children completed two blocks of testing: In the first block, they performed a childappropriate interference control task (Roebers \& Kauer, 
2009; Roebers, Schmid, \& Roderer, 2010). Based on their performance in this task (i.e., averaged reaction times for the incongruent trials in the interference control task), children were classified into one of the four quartiles. For the second testing session, children were seated in a comfortable armchair in front of the eye tracking system (Tobii 1750) hidden in a 17" computer screen. While the slight backwards inclination of the armchair reduced body movement, the instruction to sit comfortably with the head leaning on the back-rest of the chair aimed at reducing head movements. The screen was fixed on a mounting enabling individual and optimal placement of the screen $60 \mathrm{~cm}$ from the children's faces. Children were not aware of the eye tracking device. Color vision was ensured using 6 Ishihara Plates (Ishihara, 1977). Next, a five-point eye tracker calibration was done with a very simple task, while keeping the child unaware of the task's purpose. After successful calibration, children performed one of two versions of the Backward color recall task depending on experimental condition (Figure 2, see below), while their eye movements were recorded. At the end of this session, children were allowed to choose a small present.

\section{Apparatus}

The interference control task was programmed and run using E-Prime software (Psychology Software Tools, PST, Pittsburgh, PA). Accuracy and response latencies were recorded with buttons (interfaced to the computer via a serial response box) designed for use with preschool children.

The Backward color recall task was presented using the remote non-invasive eye tracking system (Tobii 1750). The LCD display of the system measured $33 \mathrm{~cm} \mathrm{x}$ $24 \mathrm{~cm}$ and the task was presented at a resolution of 1280 x 1024 pixel. Eye movements were sampled binoculary at a sampling rate of $50 \mathrm{~Hz}$. Fixation positions are identified with an accuracy of less than 1 degree and a headmovement compensation error of less than 1 degree of visual angle.

Analysis of fixation duration. The task was programmed and data were recorded with Clearview software, integrated in the Tobii 1750 device. All eye movement data falling within a predefined area with an accumulated duration of at least $100 \mathrm{~ms}$ were integrated

and counted as a fixation. To compensate for inaccuracies of the eye tracking recordings due to minor head movements as well as the large variability in the children's eye movements, this area was set at a radius of 2 degrees of visual angle. Fixations on the stimuli were identified by defining Areas of Interest (AOI) according to the location of the stimuli. In order to include all relevant fixations, this area was extended 1 degree of visual angle beyond the edge of the stimuli. The analyses of fixation time are based on the sum score of the duration of all fixations within one AOI.

\section{Tasks}

The interference control task was adapted from the Eriksen-Flanker task (Eriksen \& Eriksen, 1974), and has been used in previous studies (Roebers \& Kauer, 2009; Roebers, et al., 2010). This task requires participants to make responses based on the orientation of a central target stimulus (a fish) by pressing the corresponding button to their right or left. In the neutral condition (a third of the trials), the central target stimulus either appeared alone or with neutral adjacent flanker stimuli not implying any response (starfish). In the congruent condition, flanker stimuli consisted of fish pointing in the same direction, and in the incongruent condition, flanker stimuli pointed in the opposite direction. Each trial began with a cross-hair. The stimulus array was then presented for a maximum of 2500 milliseconds or until a response had occurred, with the interval between response and onset of the next trial varying randomly between 800 and 2000 milliseconds. The stimulus array subtended approximately $12^{\circ}$ of visual angle, with participants seated approximately $60 \mathrm{~cm}$ from the screen. Participants were instructed to feed the hungry fish in the center by pressing the button on the side where the fish's mouth was pointing to as fast and accurately as possible, while ignoring the flanker stimuli. After instruction and a practice session with 12 trials, each child completed two blocks with 24 trials each. At the end of each block, a feedback based on the accuracy of the responses was given to the child, showing how well the fish had been fed. Accuracies, as well as response latencies were recorded. Response latencies constituted the primary dependent variable since accuracy was very high in this task (mean accuracy level was $92 \%$ or above for all conditions; SD ranging from $5 \%$ to $10 \%$ ).

Figure 1 presents mean reaction time for the accurate responses as a function of condition (alone, neutral, congruent, and incongruent). It can be seen that reaction times were slowest in the trials with the highest 
interference control demands, that is, the incongruent trials. An analysis of variance with task condition as within-participant factor was conducted to document and validate the increasing need of interference control across conditions. It revealed that overall reaction times increased substantially across conditions, $\mathrm{F}(1,73)=$ 28.07, $\mathrm{p}<.001$. Analyses of variance with two conditions only further showed that reaction times were slower in the congruent compared to the neutral and alone conditions, $\mathrm{F}(1,74)=5.57, \mathrm{p}<.02$, and that there was a further significant increase in reaction time between the congruent and incongruent condition, $F(1,74)=12.69$, $p$ $<.01$. Therefore, and as has been done in other studies (e.g., Diamond, Barnett, Thomas, \& Munroe, 2007), averaged reaction times of the incongruent trials constitute the primary dependent variable for the analyses reported below.

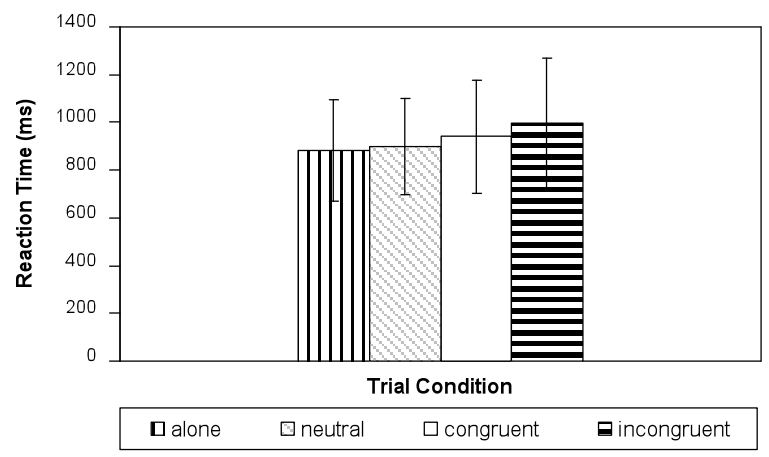

Figure 1: Mean reaction times of the four conditions of the interference control task; error bars depict standard deviations.

In its original version (condition without distractors), the Backward color recall task (Roebers \& Kauer, 2009; Schmid, Zoelch, \& Roebers, 2008; Schumann-Hengsteler et al., 2010; Hasselhorn et al., 2011) requires participants to recall a sequence of color stimuli (circles, only onesyllable color German labels), visually presented for 1000 milliseconds, in reversed order. Figure 2 illustrates the sequence and the stimuli of this task (with distractors, see below). The cover story comprised a walking dwarf who looses discs out of his bag and consequently has to turn around and collect them (in reversed order). After instruction and three practice trials, testing began with 6 trials at a list length of two colors. Having completed these trials, the number of items was increased by one. If more than half of the trials at any list length were incorrectly recalled, the task ended after completion of all trials of the respective list. Participants' scores were computed as the total sum of correctly recalled trials.

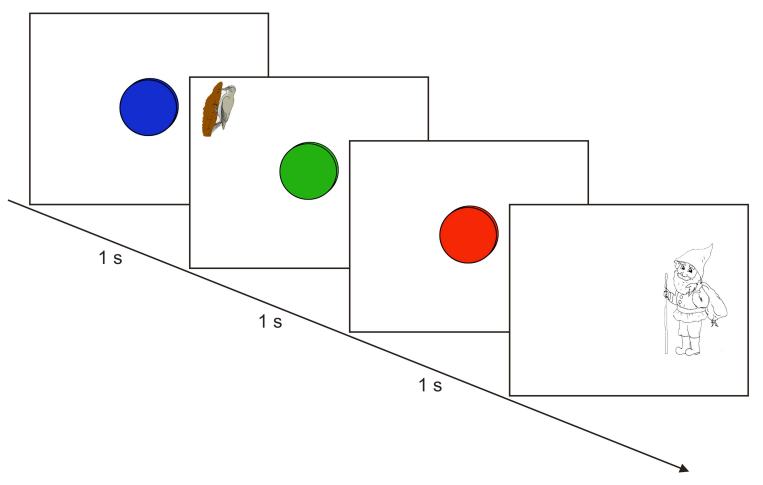

Figure 2: Sequence of a trial of the Backward Color Recall Task in the condition with distracting stimuli appearing on the array

In the condition with distractors, administration and scoring of the Backward color recall task was exactly the same, with the exception that distractor stimuli (animals and objects that can be encountered in a forest) appeared on the screen while the color stimuli were presented (see Figure 2). During each trial, one of the target color stimuli was presented together with a peripheral distractor stimulus, appearing in one of four positions on the screen (right upper corner, right bottom corner, left upper corner, left bottom corner). Positions of the distractors on the screen and occurrence in time sequence were counterbalanced. Prior to practice trials, children were shown the distracting stimuli on a sheet of paper and given time to look at them. In addition, they were explicitly told not to attend to these distractors during the task's course.

\section{Results}

Since initial analyses did not reveal significant and consistent sex differences, data of boys and girls were collapsed for further analyses.

Descriptive statistics for performance on the interference control task are provided in Table 2. As can be seen, the groups differed substantially in terms of their interference control skills as was intended by the quartile grouping. More importantly, however, Table 2 reveals that there were no a priori differences in interference 
control skills between the two experimental conditions that might contaminate the subsequent analysis $(F<1)$. Further, no interaction between group and condition $(F<$ 1) was found, confirming that across the two experimental conditions children did not differ with respect to their interference control skills, that is, matching was successful.

Table 2: Group comparisions of the mean reaction times (in msec; standard deviations in parenthesis) in the incongruent trials of the interference control task as a function of experimental condition

\begin{tabular}{rcc}
\hline & $\begin{array}{c}\text { WM Trials without } \\
\text { Distractor }\end{array}$ & $\begin{array}{c}\text { WM Trials with } \\
\text { Distractors }\end{array}$ \\
\hline Low & $1364(80)$ & $1426(85)$ \\
Medium low & $1040(40)$ & $1041(58)$ \\
Medium high & $867(52)$ & $853(46)$ \\
High & $713(70)$ & $712(73)$ \\
\hline
\end{tabular}

For performance of the Backward color recall task as a function of interference control skills, an ANOVA with between-subjects groups based on quartiles of the distribution in interference control (that is, a high performing group, two medium performing groups, and a low performing group) was conducted. Main effects of experimental condition, $F(1,67)=10.98, p<.01$, partial $\eta^{2}=.14$, and of interference control group, $F(3,67)=$ 2.97, $p<.05$, partial $\eta^{2}=.12$, but no interaction were found. As Figure 3 illustrates, working memory performance in the condition with distractors was overall poorer compared to the condition without distractors.

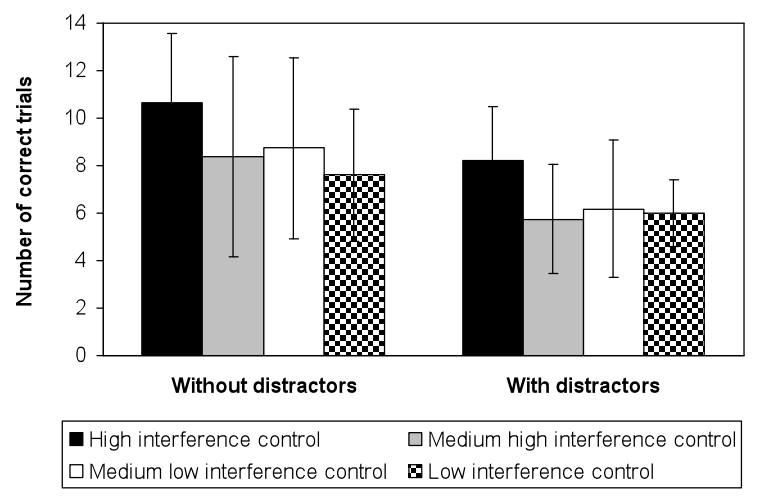

Figure 3. Mean number of correct trials in the Backward color recall task as a function of interference control group (quartiles) and experimental condition. Error bars depict standard deviations.
And, children's performance of the Backward color recall task differed depending on their interference control skills. Post hoc tests following the StudentNewman-Keuls procedure (with Bonferroni-corrections for multiple comparisons) revealed that only two groups differed significantly, that is, children displaying high interference control performed significantly better than children with low interference control $(t(35)=2.88, p<$ $.01, d=0.95$ ) (see Figure 3).

Descriptive statistics for fixation time on targets (and distractors) for children in the experimental condition with distractors are displayed in Table 3. A two-way mixed-design ANOVA with distractor presence (target presented with or without distractor) and interference control group (high, medium high, medium low vs. low performing children) as a between-subjects factors was performed on mean fixation time on target stimuli, revealing a highly significant effect of distractor presence, $F(1,33)=300.93, p<.001$, partial $\eta^{2}=.90$, indicating that children spent less time looking at target stimuli if a distractor was present compared to when no distractor appeared. There was neither a significant effect of interference control group nor an interaction between distractor presence and interference control group $(F<$ 1), implying that children with higher or lower interference control skills did not differ in their pattern of looking at the target stimuli. Moreover and as can be seen in Table 3, the four groups did also not differ with respect to their fixation time on distractors $(F<1)$.

Table 3: Mean gaze time on targets and on distractors as a function of interference control group and of stimuli (stimuli of the working memory task presented with or without distractors; condition with distractors only)

\begin{tabular}{rccc}
\hline & \multicolumn{2}{c}{ With Distractors } & Without Distractors \\
& $\begin{array}{c}\text { Gaze Time on } \\
\text { Targets }\end{array}$ & $\begin{array}{c}\text { Gaze Time on } \\
\text { Distractors }\end{array}$ & $\begin{array}{c}\text { Gaze Time on } \\
\text { Targets }\end{array}$ \\
\hline Interference Control & & & \\
Lew & $466(129)$ & $319(131)$ & $814(80)$ \\
Medium low & $486(167)$ & $282(105)$ & $804(83)$ \\
High & $488(132)$ & $310(138)$ & $805(99)$ \\
\hline
\end{tabular}




\section{Discussion}

The major aim of the present study was to investigate the role of interference control processes for working memory performance. While there is relatively consistent empirical evidence for an association between working memory and interference control in adults and although most of the current theoretical frameworks of working memory emphasize attentional processes (Baddeley, 2001; Cowan, 2005; Kane \& Engle, 2000, 2003), little is known about this relationship in young children. Therefore, we investigated the role of interference control for 6-year-olds' working memory performance (a) by means of grouping children into four groups based on quartiles of interference control and subsequently comparing their working memory performance, (b) by manipulating the need to control interference within the task and assess the detrimental effect on working memory performance, and (c) by assessing visual attention during presentation of the to-be-remembered stimuli through eye-tracking to get insights to attention allocation and its role for working memory performance.

When comparing children with high, medium high, medium low, and low interference control skills, systematic differences in working memory performance were found: participants with superior interference control skills outperformed age mates with less welldeveloped interference control skills. This finding can be interpreted as showing that more advanced interference control skills imply an advantage for working memory performance and supports the assumption that interference control is conceptually distinguishable from working memory. Whether the advantage of high interference control skills would also generalize to other cognitive operations and domains remains to be shown. For working memory, however, the present results confirm a relationship between the two constructs and allow to generalize current conceptualizations of working memory also to young children (Cowan, 2005; Kane \& Engle, 2003).

Interestingly, substantial working memory differences only emerged between the high and low interference control groups. Figure 3 nicely illustrates that only children with pronounced and well-developed interference control skills showed superior working memory performance compared to the groups with lower interference control skills. This pattern was found in both conditions, that is, in tasks with and without distractors increasing the reliability of this finding. Inspection of performance seems to suggest that the impact of interference control on working memory is not linear; rather, only pronounced differences in interference control skills seem to carry over into working memory performance differences. Although this interpretation is to be treated with cautious, this specific pattern might show that quantitative differences in basic interference control abilities fuel qualitative differences when a certain level of quantitative differences is reached. This preliminary and somewhat speculative explanation is, however, in line with current theories of ontogenetic development combining stage-like theories (e.g., Piaget) with neo-piagetian information processing approaches to human development (Bjorklund, 2005). Alternatively, one might assume that general cognitive skills are an underlying factor contributing to both poorer interference control and working memory. Further research, however, is certainly necessary.

With regard to performance on the working memory task, increasing the need to control interference stemming from the simultaneous presentation of distracting items confirmed the assumed relation between interference control and working memory. Overall, performance on the Backward color recall task was poorer when distracting stimuli were presented simultaneously. Since visual attention was quantified by recording fixations, we can be sure that participants did look at the distractors. The decrease in correctly recalled trials under distracting conditions can thus be interpreted as showing that processes of interference control are directly involved in the mastery of this working memory task. Together with the findings from the individual differences approach, the observed working memory impairments in the condition with distractors offer converging evidence for a multifaceted (indirect and direct) relation between interference control on working memory.

There was no interaction between presence of a distractor and interference control abilities. Moreover, gaze time of the four groups with differing interference control skills did not vary systematically. At first sight, these findings may be surprising because low interference control skills should further decrease working memory performance under strong needs of interference control. And, low interference control skills should have an negative and proximal impact on interference control while mastering a working memory task. One possible 
interpretation of this counterintuitive finding may be that although children did look at the distractors, as proven by the gaze time allocation, this information was not processed or did not demand attentional processes. Such an interpretion would - at least to some extent - imply that eye-tracking data does not solely measure visual attention. More research in this respect is clearly necessary.

Alternatively, previous work has shown that there are distinguishable aspects of interference control within its theoretical framework and when measured separately, distinct patterns of interrelations with task performance may emerge (e.g., Ridderinkhof, van der Moolen, \& Band, 1997; Roebers et al., 2010). Controlling, adapting, preparing, executing and inhibiting motor responses may be empirically and theoretically have to be distinguish from higher-order attentional processes, also belonging to the concept of interference control. Our eye-tracking data may point to a differentiated picture of the underlying processes involved in the relation between interference control and working memory suggesting that different aspects of interference control may get activated depending on the cognitive activity and the cognitive processes being called for. Certainly, our results also imply a certain dependency of assessment method and the power analysis revealed that there was not enough power in the data to reliably detect an interaction. Our interpretation should therefore treated with cautious.

The present approach of measuring visual attention towards targets and distractors allowed estimating its impact on performance. And, the classifications of children into groups of high, medium high, medium low, and low interference control skills resulted in systematic differences in working memory performance, a psychological construct of great importance for many higher- order cognitive functions. For young children, based on additional empirical evidence, poor interference control skills may ultimatively be considered as a behavioral "marker" (Goswami, 2009) of a risk of poor working memory development, increasing the risk for deficits in school readiness and for school failure. Although further research is clearly necessary, the present study offers intriguing insights into the relationship between interference control and working memory and the conditions under which it applies.

Taken together, the present study presents converging evidence that interference control processes play a substantial role for working memory performance. At the same time, we are aware that our findings may not perfectly generalize to other operationalizations of interference control and working memory. Nevertheless, we are convinced that these results mark a starting point for future research with different (older and younger) age groups and new methods in order to complete the picture of how not only interference control skills, but also online interference control during a learning task, measured with eye tracking, impact actual task performance.

\section{Acknowledgments}

The work presented in this paper was financially supported by a grant to the first author from the Swiss National Science Foundation (SNF- Grant No. 100014112492). We wish to thank the kindergarten teachers and children for their cooperation, the Tobii team for their technical support, and Jonas Schmid for his help with the data collection.

\section{References}

Alloway, T. P. (2007). Working memory, reading, and mathematical skills in children with developmental coordination disorder. Journal of Experimental Child Psychology, 96, 20-36.

Alloway, T. P., Gathercole, S. E., Willis, C., \& Adams, A.-M. (2004). A structural analysis of working memory and related cognitive skills in young children. Journal of Experimental Child Psychology, 87, 85-106.

Awh, E., \& Jonides, J. (2001). Overlapping mechanisms of attention and working memory. Trends in Cognitive Sciences, 5, 119-126.

Baddeley, A. D. (2001). Is working memory still working? American Psychologist, 56, 851-864.

Bayliss, D. M., Jarrold, C., Gunn, D. M., \& Baddeley, A. D. (2003). The complexities of complex span: Explaining individual differences in working memory in children and adults. Journal of Experimental Psychology: General, 132, 71-92.

Best, J. R., Miller, P. H., \& Jones, L. L. (2009). Executive functions after age 5: Changes and correlates. Developmental Review, 29, 180-200. 
Bull, R., Johnston, R. S., \& Roy, J. A. (1999). Exploring the roles of the visual-spatial sketch pad and central executive in children's arithmetical skills: Views from cognition and developmental neuropsychology. Developmental Neuropsychology, 15, 421-442.

Bjorklund D. F. (2005). Children's thinking: Cognitive development and individual differences, $\left(4^{\text {th }} \mathrm{ed}\right.$.). Thomson Learning, Inc, Belmont, CA

Cowan, N. (2005). Working memory capacity. Hove, East Sussex, UK: Psychology Press.

Cowan, N., \& Alloway, T. (2009). Development of Working Memory In Childhood. In M.L. Courage \& $\mathrm{N}$. Cowan (Eds.), The development of memory in infancy and childhood. Hove, East Sussex, UK: Psychology Press, 303-342.

Cowan, N., Elliott, E. M., Saults, J. S., Morey, C. C., Mattox, S., Hismjatullina, A., \& Conway, A.R.A. (2005). On the capacity of attention: Its estimation and its role in working memory and cognitive aptitudes. Cognitive Psychology, 51, 42-100.

Daneman, M., \& Merikle, P. M. (1996). Working memory and language comprehension: A metaanalysis. Psychonomic Bulletin \& Review, 3, 422-433.

Davidson, M. C., Amso, D., Anderson, L. C., \& Diamond, A. (2006). Development of cognitive control and executive functions from 4 to 13 years: Evidence from manipulations of memory, inhibition, and task switching. Neuropsychologia, 44, 2037-2078.

Diamond, A., Barnett, W. S., Thomas, J., \& Munro, S. (2007). Preschool program improves cognitive control. [Journal; Peer Reviewed Journal]. Science, 318, 1387-1388.

Engle, R. W., Tuholski, S. W., Laughlin, J. E., \& Conway, A. R. A. (1999). Working memory, shortterm memory, and general fluid intelligence: A latentvariable approach. Journal of Experimental Psychology: General, 128, 309-331.

Eriksen, B. A., \& Eriksen, C. W. (1974). Effects of noise letters upon the identification of a target letter in a nonsearch task. Perception and Psychophysics, 16, 143-149.

Faul, F., Erdfelder, E., Lang, A.-G., \& Buchner, A. (2007). G*power 3: A flexible statistical power analysis program for the social, behavioral, and biomedical sciences. Behavior Research Methods, 39, 175-191.
Garon, N., Bryson, S. E., \& Smith, I. M. (2008). Executive function in preschoolers: A review using an integrative framework. Psychological Bulletin, 134, 31-60.

Gathercole, S. E., Alloway, T. P., Willis, C., \& Adams, A.-M. (2006). Working memory in children with reading disabilities. Journal of Experimental Child Psychology, 93, 265-281.

Gathercole, S. E. \& Baddeley, A. D. (1993). Working Memory and Language. Hove, UK: Lawrence Erlbaum.

Goswami, U. (2009). Mind, brain, and literacy: biomarkers as usable knowledge for education. Mind, Brain, and Education, 3, 176-184.

Hasselhorn, M., Schumann-Hengsteler, R., Gronauer, J., Grube, D., Mähler, C., Schmid, I., Seitz-Stein, K., \& Zoelch, C. (2011). Arbeitsgedächtnistestbatterie für Kinder von 5 bis 12 Jahren (AGTB 5-12). Göttingen: Hogrefe.

Hollingworth, A., \& Henderson, J. M. (2002). Accurate visual memory for previously attended objects in natural scenes. Journal of Experimental Psychology: Human Perception and Performance, 28, 113-136.

Ishihara, S. (1977). Tests for colour-blindness, 24 plates ed. Tokyo: Kanehara Shuppan.

Kail, R., \& Hall, L. K. (2001). Distinguishing short-term memory from working memory. Memory \& Cognition, 29, 1-9.

Kane, M. J., Conway, A. R. A., Hambrick, D. Z., \& Engle, R. W. (2008). Variation in working memory capacity as variation in executive attention and control. In A. R. A. Conway \& C. Jarrold \& M. J. Kane \& A. Miyake \& J. N. Towse (Eds.), Variation in Working Memory (pp. 22-48). New York: Oxford University Press.

Kane, M. J., \& Engle, R. W. (2000). Working-memory capacity, proactive interference, and divided attention: Limits on long-term memory retrieval. Journal of Experimental Psychology: Learning, Memory and Cognition, 26, 336-358.

Kane, M. J., \& Engle, R. W. (2003). Working-memory capacity and the control of attention: The contributions of goal neglect, response competition, and task set to Stroop interference. Journal of Experimental Psychology: General, 132, 47-70. 
Karatekin, C. (2007). Eye tracking studies of normative and atypical development. Developmental Review, 27, 283- 348.

Kyllonen, P. C., \& Christal, R. E. (1990). Reasoning ability is (little more than) working-memory capacity? Intelligence, 14, 389-433.

Lee, K., Ng, E. L., \& Ng, S. F. (2009). The contributions of working memory and executive functioning to problem representation and solution generation in algebraic word problems. Journal of Educational Psychology, 101, 373-387.

Marcovitch, S., Boseovski, J. J., \& Knapp, R. J. (2007). Use it or lose it: Examining preschoolers' difficulty in maintaining and executing a goal. Developmental Science, $10,559-564$.

Pickering, S. J., \& Gathercole, S. E. (2001). Working Memory Test Battery for Children (WMTB-C). London: The Psychological Corporation.

Roebers, C. M. \& Kauer, M. (2009). Motor and Cognitive Control in a Normative Sample of 7 Year Olds. Developmental Science, 12, 175- 181.

Roebers, C. M., Schmid, C., \& Roderer, T. (2010). Encoding strategies in primary school children: Insights from an eye-tracking approach and the role of individual differences in attentional control. The Journal of Genetic Psychology, 17, 1-21.

Ridderinkhof, K. R., van der Molen, M. W., \& Band, P. H. (1997). Sources of interference from irrelevant information: A developmental study. Journal of Experimental Child Psychology, 65, 315-341.

Rueda, M., R., Rothbart, M. K., McCandliss, B. D., Saccomanno, L., \& Posner, M. I. (2005). Training, maturation, and genetic influences on the development of executive attention. Proceedings of the National Academy of Sciences of the United States of America, 11, 14931-14936.

Schmid, C., Zoelch, C., \& Roebers, C. (2008). Das Arbeitsgedächtnis von 4- bis 5-jährigen Kindern: Theoretische und empirische Analyse seiner Funktionen. Zeitschrift für Entwicklungspsychologie und Pädagogische Psychologie, 40, 2-12.
Schumann-Hengsteler, R., Grube, D., Zoelch, C., Mähler, C., Seitz-Stein, K., Schmid, I., Gronauer, J., \& Hasselhorn, M. (2010). Differentialdiagnostik der Funktionstüchtigkeit des Arbeitsgedächtnisses bei Kindern mit der AGTB 5-12. In H.-P. Trolldenier, W. Lenhard, \& P. Marx (Eds.), Brennpunkte der Gedächtnisforschung: Entwicklungs- und pädagogisch-psychologische Perspektiven (pp.305319). Göttingen: Hogrefe.

St Clair-Thompson, H., \& Gathercole, S. E. (2006). Executive functions and achievements in school: Shifting, updating, inhibition, and working memory. The Quarterly Journal of Experimental Psychology, 59, 745-759.

Swanson, L., \& Kim, K. (2007). Working memory, shortterm memory, and naming speed as predictors of children's mathematical performance. Intelligence, 35, 151-168.

Thorell, L. B., Lindqvist, S., Nutley, S. B., Bohlin, G., \& Klingberg, T. (2009). Training and transfer effects of executive functions in preschool children. Developmental Science, 12, 106-113.

Towse, J. N., Hitch, G. J., \& Hutton, U. (1998). A reevaluation of working memory capacity in children. Journal of Memory and Language, 39, 195-217.

Võ, M. L.-H., Schneider, W. X., \& Matthias, E. (2008). Transsaccadic Scene Memory Revisited: A 'Theory of Visual Attention (TVA)' based Approach to Recognition Memory and Confidence for Objects in Naturalistic Scenes. Journal of Eye-Movement Research, 2, 1-13.

Wedel, M., Pieters, R., \& Liechty, J. (2008). Attention switching during scene perception: How goals influence the time course of attention across advertisements. Journal of Experimental Psychology: Applied, 14, 129-138.

Wienrich, C., Hesse, U. \& Müller-Plath, G. (2009). Eye movements and attention in visual feature search with graded target-distractor-similarity. Journal of Eye Movement Research, 3, 1-19. 